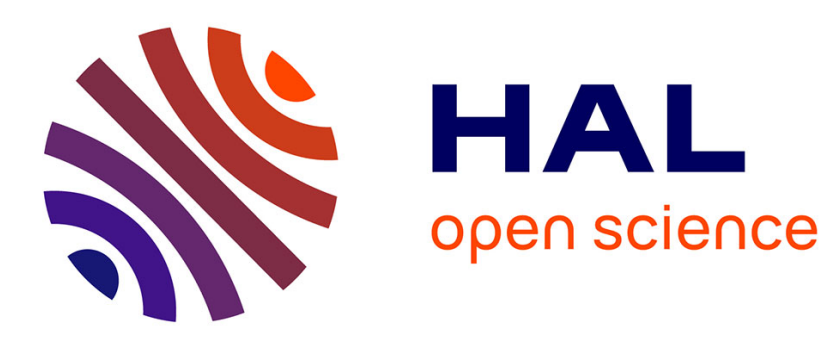

\title{
Error studies of the Coupling Darcy-Stokes system with velocity-pressure formulation
}

\author{
Fida El Chami, Gihane Mansour, Toni Sayah
}

\section{To cite this version:}

Fida El Chami, Gihane Mansour, Toni Sayah. Error studies of the Coupling Darcy-Stokes system with velocity-pressure formulation. 2011. hal-00511792v3

\section{HAL Id: hal-00511792 \\ https://hal.science/hal-00511792v3}

Preprint submitted on 27 Apr 2011

HAL is a multi-disciplinary open access archive for the deposit and dissemination of scientific research documents, whether they are published or not. The documents may come from teaching and research institutions in France or abroad, or from public or private research centers.
L'archive ouverte pluridisciplinaire HAL, est destinée au dépôt et à la diffusion de documents scientifiques de niveau recherche, publiés ou non, émanant des établissements d'enseignement et de recherche français ou étrangers, des laboratoires publics ou privés. 


\title{
ERROR STUDIES OF THE COUPLING DARCY-STOKES SYSTEM WITH VELOCITY-PRESSURE FORMULATION
}

\author{
FIDA EL CHAMI ${ }^{\dagger}$, GIHANE MANSOUR $^{\ddagger}$ AND TONI SAYAH
}

\begin{abstract}
In this paper we study the Coupling Darcy-Stokes Systems. We establish a coupled variational formulation with the velocity and the pressure. The velocity is approximated with curl conforming finite elements and the pressure with standard continuous elements. We establish optimal a priori and a posteriori error estimates. We conclude our paper with some numerical simulations.
\end{abstract}

Keywords Stokes equations, Darcy equations, a piori and a posteriori errors.

\section{InTRODUCTION.}

The numerical simulation of underground flows can be treated as cracks in porous media. Indeed, the flow of a viscous incompressible fluid in a porous medium is usually modelled by Darcy equations and, when the thickness of the crack is too large to be neglected, the Stokes system must be considered in the crack and coupled with these equations. In this work, we consider the following system already studied in [4], [5] and [6]:

Let $\Omega$ and $\Omega_{F}$ be bounded connected open domains in $\mathbb{R}^{3}$ with Lipschitz-continuous boundaries, such that $\bar{\Omega}_{F}$ is contained in $\Omega$. For simplicity, we also assume that $\Omega_{F}$ is simply connected and has a connected boundary. We set $\Omega_{P}=\Omega \backslash \bar{\Omega}_{F}$ and we denote by $\Gamma=\partial \Omega_{F}$ the interface between $\Omega_{F}$ and $\Omega_{P}$. Let also $\mathbf{n}$ stand for the unit outward normal vector to $\Omega_{P}$ on its boundary $\partial \Omega_{P}$.

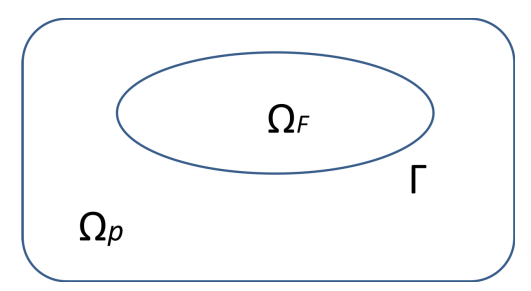

Figure 1. The geometry

April 19, 2011.

† Faculté des Sciences II, Université Libanaise, Département de mathématiques, B.P. 90656, Fanar-Maten, Liban.

¥ Faculté des Sciences, Université Saint-Joseph, B.P 11-514 Riad El Solh, Beyrouth 1107 2050, Liban. 
We consider the following system of equations:

$$
(P) \begin{cases}\mu \mathbf{u}+\nabla p=\mathbf{f} & \text { in } \Omega_{P} \\ -\nu \Delta \mathbf{u}+\nabla p=\mathbf{f} & \text { in } \Omega_{F} \\ \operatorname{div} \mathbf{u}=0 & \text { in } \Omega_{P} \cup \Omega_{F} \\ \mathbf{u} \cdot \mathbf{n}=0 & \text { on } \partial \Omega \\ \left(\mathbf{u}_{\Omega_{P}}-\mathbf{u}_{\Omega_{F}}\right) \cdot \mathbf{n}=0 & \text { on } \Gamma \\ p_{\left.\right|_{\Omega_{P}}}-p_{\left.\right|_{\Omega_{F}}}=0 & \text { on } \Gamma \\ \mathbf{c u r l} \mathbf{u}_{\Omega_{F}} \times \mathbf{n}=0 & \text { on } \Gamma\end{cases}
$$

where $\mathbf{u}$ is the velocity, $p$ the pressure, $\mathbf{f}$ the density of body forces and $\nu$ and $\mu$ positive constants.

For the Vorticity-velocity-pressure formulation for the Stokes problem, we refer to [10], [11] and [20]. For the coupling problem, we cite the works [2], [3], [8], [9], [16] and [21]. In [1] and [12], we treat the Stokes problem with non-standard boundary conditions and we introduce velocity-pressure weak formulation. In [4], [5] and [6], the basic idea consists in introducing the vorticity $\mathbf{w}=$ curl $\mathbf{u}$ as a new unknown on the fluid domain $\Omega_{F}$. However, we treat in this work the same systems with the unknowns $\mathbf{u}$ and $p$ without introducing the vorticity $\mathbf{w}$. Since, we can discretize the pressure and the velocity independently without a discrete inf-sup condition to obtain matrix systems with an optimal dimension and optimal time of resolution.

The outline of the paper is as follows:

- In Section 2, we introduce the problem, establish a decoupled variational formulation and prove its wellposedness.

- In section 3, we introduce the finite elements and a fully discrete system using the curl conforming finite elements for the velocity and the standard continuous elements for the pressure.

- We prove the a priori and a posteriori estimates in sections 4 and 5 , respectively.

- Section 6 is devoted to numerical experiments wich confirm the theoretical results.

\section{AnAlysis OF THE MODEL}

In all the paper, we suppose that $\mathbf{f} \in L^{2}(\Omega)^{3}$ and we denote by $C$ and $c$ generic positive constants.

In order to write the variational formulation of the previous problem, we introduce the following spaces:

$$
\begin{aligned}
& W^{m, p}(\Omega)=\left\{\mathbf{v} \in L^{p}(\Omega)^{3}, \partial^{\alpha} \mathbf{v} \in L^{p}(\Omega)^{3}, \forall|\alpha| \leq m\right\}, \\
& H^{m}(\Omega)=W^{m, 2}(\Omega),
\end{aligned}
$$

equipped with the undermentionned semi-norm and norm:

$$
|\mathbf{v}|_{m, p, \Omega}=\left(\sum_{|\alpha|=m} \int_{\Omega}\left|\partial^{\alpha} \mathbf{v}(x)\right|^{p} d x\right)^{1 / p} \quad \text { and } \quad\|\mathbf{v}\|_{m, p, \Omega}=\left(\sum_{k \leq m}|\mathbf{v}|_{k, p, \Omega}^{p}\right)^{1 / p}
$$

As usual, we shall omit $p$ when $p=2$ and denote by $(\cdot, \cdot)$ the scalar product of $L^{2}(\Omega)$. Also, recall the familiar notation:

with the Poincaré inequality

$$
H_{0}^{1}(\Omega)=\left\{\mathbf{v} \in H^{1}(\Omega) ; \mathbf{v}=0 \text { on } \partial \Omega\right\}
$$

$$
\forall \mathbf{v} \in H_{0}^{1}(\Omega),\|\mathbf{v}\|_{0, \Omega} \leq C|\mathbf{v}|_{1, \Omega}
$$


Finally, we introduce the spaces:

$$
\begin{aligned}
& H(\operatorname{div}, \Omega)=\left\{\mathbf{v} \in L^{2}(\Omega)^{3}, \operatorname{div} \mathbf{v} \in L^{2}(\Omega)\right\}, \quad H_{0}(\operatorname{div}, \Omega)=\{\mathbf{v} \in H(\operatorname{div}, \Omega), \mathbf{v} \cdot \mathbf{n}=0 \text { on } \partial \Omega\}, \\
& H(\mathbf{c u r l}, \Omega)=\left\{\mathbf{v} \in L^{2}(\Omega)^{3}, \mathbf{c u r l} \mathbf{v} \in L^{2}(\Omega)^{3}\right\}, \quad H_{0}(\mathbf{c u r l}, \Omega)=\{\mathbf{v} \in H(\mathbf{c u r l}, \Omega), \mathbf{v} \times \mathbf{n}=0 \text { on } \partial \Omega\},
\end{aligned}
$$

normed respectively by:

$$
\|\mathbf{v}\|_{H(\operatorname{div}, \Omega)}=\left(\|\mathbf{v}\|_{0, \Omega}^{2}+\|\operatorname{div} \mathbf{v}\|_{0, \Omega}^{2}\right)^{1 / 2},
$$

and

$$
\|\mathbf{v}\|_{H(\operatorname{curl}, \Omega)}=\left(\|\mathbf{v}\|_{0, \Omega}^{2}+\|\operatorname{curl} \mathbf{v}\|_{0, \Omega}^{2}\right)^{1 / 2}
$$

We recall that the trace operator: $\mathbf{v} \rightarrow \mathbf{v . n}$ is continuous from $H(\operatorname{div}, \Omega)$ onto $H^{-1 / 2}(\partial \Omega)$ and the jump $\left(\left.\mathbf{v}\right|_{\Omega_{P}}-\left.\mathbf{v}\right|_{\Omega_{F}}\right) . \mathbf{n}$ vanishes on $\Gamma$.

We recall an important lemma which will be useful in the rest of the paper:

Lemma 2.1. There exists a unique solution $\mathbf{w}$ in $H^{1}(\Omega) / \mathbb{R}$ such that

$$
(\nabla \mathbf{w}, \nabla \mathbf{v})=(\mathbf{f}, \nabla \mathbf{v}), \forall \mathbf{v} \in H^{1}(\Omega) / \mathbb{R},
$$

and there exists a positive constant $C$ such that

$$
\|\mathbf{w}\|_{1, \Omega} \leq C\|\mathbf{f}\|_{0, \Omega} .
$$

For the following regularity theorem, we refer to Nedelec [18].

Theorem 2.2. All functions $\mathbf{v} \in L^{2}\left(\Omega_{F}\right)^{3}$ satisfying:

$$
\operatorname{div} \mathbf{v}=0, \quad \operatorname{curl} \mathbf{v} \in L^{2}\left(\Omega_{F}\right)^{3}, \quad \mathbf{v} \cdot \mathbf{n}=0 \text { on } \Gamma,
$$

verify

$$
\|\mathbf{v}\|_{0, \Omega_{F}} \leq C\|\mathbf{c u r l} \mathbf{v}\|_{0, \Omega_{F}}
$$

If $\Omega_{F}$ is convex, $\mathbf{v}$ belongs to $H^{1}\left(\Omega_{F}\right)$ and we have

$$
\|\mathbf{v}\|_{1, \Omega_{F}} \leq C\|\mathbf{c u r l} \mathbf{v}\|_{0, \Omega_{F}} .
$$

We have also from [4] (page 10 lemma 2.2) the next lemma:

Lemma 2.3. For all functions $\mathbf{v} \in L^{2}(\Omega)^{3}$ satisfying:

$$
\operatorname{curl} \mathbf{v}_{\left.\right|_{\Omega_{F}}} \in L^{2}\left(\Omega_{F}\right)^{3}, \operatorname{div} \mathbf{v}=0 \text { in } \Omega, \mathbf{v} \cdot \mathbf{n}=0 \text { on } \partial \Omega
$$

there exist a constant $\alpha_{0}$ such that:

$$
\|\mathbf{v}\|_{0, \Omega_{F}} \leq \alpha_{0}\left(\|\mathbf{v}\|_{0, \Omega_{P}}^{2}+\|\operatorname{curl} \mathbf{v}\|_{0, \Omega_{F}}^{2}\right)^{1 / 2}
$$

In order to give the variational formulation of the problem $(P)$, we introduce the space:

$$
X=\left\{\mathbf{v} \in L^{2}(\Omega)^{3}, \operatorname{curl} \mathbf{v}_{\left.\right|_{\Omega_{F}}} \in L^{2}\left(\Omega_{F}\right)^{3}\right\},
$$

provided with the norm

$$
\|\mathbf{v}\|_{X}=\left(\|\mathbf{v}\|_{0, \Omega}^{2}+\|\operatorname{curl} \mathbf{v}\|_{0, \Omega_{F}}^{2}\right)^{1 / 2}
$$

We consider the following weak variational formulation, denoted by $(V)$ :

Find $\mathbf{u} \in X$ and $p \in H^{1}(\Omega) / \mathbb{R}$ such that

$$
\begin{array}{r}
\mu(\mathbf{u}, \mathbf{v})_{\Omega_{P}}+\nu(\mathbf{c u r l} \mathbf{u}, \mathbf{c u r l} \mathbf{v})_{\Omega_{F}}+(\nabla p, \mathbf{v})=(\mathbf{f}, \mathbf{v}), \quad \forall \mathbf{v} \in X \\
(\nabla q, \mathbf{u})=0, \quad \forall q \in H^{1}(\Omega) / \mathbb{R}
\end{array}
$$


Theorem 2.4. The problem $(P)$ is equivalent to the weak variational formulation $(V)$.

Proof: Suppose that $(\mathbf{u}, p) \in X \times H^{1}(\Omega) / \mathbb{R}$ is a solution of $(V)$.

The equation (2.3) gives the third equation of $(P)$ in the distribution sense. Since $\mathcal{D}\left(\Omega_{P}\right) \subset X$ and $\mathcal{D}\left(\Omega_{F}\right) \subset X$, we deduce from $(2.2)$ and the relation $-\Delta \mathbf{u}=\mathbf{c u r l}$ curl $\mathbf{u}$ (as we have $\left.\operatorname{div} \mathbf{u}=0\right)$ the first and the second equation of $(P)$. On the other hand, $\mathbf{u} \in H(\operatorname{div}, \Omega)$ and $(\mathbf{u}, \nabla q)=0$ for all $q \in H^{1}(\Omega)$ gives us $\left(\mathbf{u}_{\left.\right|_{\Omega_{P}}}-\mathbf{u}_{\left.\right|_{\Omega_{F}}}\right)$.n $=0$ on $\Gamma$ and $\mathbf{u . n}=0$ in $H^{-1 / 2}(\partial \Omega)$. Since $p \in H^{1}(\Omega) / \mathbb{R}$ we have $\left(p_{\left.\right|_{\Omega_{P}}}-p_{\left.\right|_{\Omega_{F}}}\right)_{\left.\right|_{\Gamma}}=0$. Finally, the second equation of $(P)$ and the equation (2.2) gives

$(\operatorname{curl} \mathbf{c u r l} \mathbf{u}, \mathbf{v})_{\Omega_{F}}=(\operatorname{curl} \mathbf{u}, \operatorname{curl} \mathbf{v})_{\Omega_{F}}, \quad \forall \mathbf{v} \in H\left(\operatorname{curl}, \Omega_{F}\right)$,

which leads to the last condition curl $\mathbf{u}_{\left.\right|_{\Omega_{F}}} \times \mathbf{n}=0$ on $\Gamma$.

Inversely, let $(\mathbf{u}, p) \in X \times H^{1}(\Omega) / \mathbb{R}$ be a solution of $(P)$. Multiplying the first equation of $(P)$ by $\mathbf{v} \in X$ and integrating over $\Omega_{F}$ and using the Green formula, and the second equation by $\mathbf{v}$ and integrating over $\Omega_{P}$ gives the first equation of $(V)$. The next three equations of $(P)$ give the second equation of $(V)$.

The variational formulation $(V)$ can be splitted into a system for the velocity and a Poisson equation for the pressure. Let us introduce the space

$$
U=\left\{\mathbf{v} \in X ;(\nabla q, \mathbf{v})=0, \forall q \in H^{1}(\Omega) / \mathbb{R}\right\} .
$$

and remark that every $\mathbf{v} \in U$ verify the lemma 2.3 .

The lemmas 2.1 and 2.3 allow us to establish the following theorem:

Theorem 2.5. The problem $(V)$ is equivalent to the problem

Find $\mathbf{u} \in U$ such that

$$
\mu(\mathbf{u}, \mathbf{v})_{\Omega_{P}}+\nu(\operatorname{curl} \mathbf{u}, \mathbf{c u r l} \mathbf{v})_{\Omega_{F}}=(\mathbf{f}, \mathbf{v}), \quad \forall \mathbf{v} \in U
$$

Find $p \in H^{1}(\Omega) / \mathbb{R}$ such that

$$
\mu(\mathbf{u}, \nabla q)_{\Omega_{P}}+(\nabla p, \nabla q)=(\mathbf{f}, \nabla q), \quad \forall q \in H^{1}(\Omega) / \mathbb{R} .
$$

Furthermore, there exists a unique solution and we have the following bounds

$$
\begin{gathered}
\|\mathbf{u}\|_{0, \Omega_{P}}+\|\mathbf{c u r l} \mathbf{u}\|_{0, \Omega_{F}} \leq C_{1}\|\mathbf{f}\|_{0, \Omega} \\
|p|_{1, \Omega} \leq C_{2}\|\mathbf{f}\|_{0, \Omega}
\end{gathered}
$$

Proof: The equivalence of the two problems comes from the fact that every element $\mathbf{v} \in X$ can be written as $\mathbf{v}=\mathbf{w}+\nabla q$ where $\mathbf{w} \in U$ and $q \in H^{1}(\Omega) / \mathbb{R}$.

The Lax-Milgram theorem gives the existence and the uniqueness of the solution of (2.4). Having the velocity, the Lax-Milgram theorem gives the existence and the uniqueness of the solution of (2.5). We obtain the inequalities by first taking $\mathbf{v}=\mathbf{u}$ in the equation (2.4), next by taking $q=p$ in the equation (2.5).

We denote by $\left(V_{1}\right)$ the problem defined by (2.4) and (2.5). Then, it is easy to show that $\left(V_{1}\right)$ is equivalent to the following problem denoted $\left(V_{2}\right)$ :

Find $\mathbf{u} \in X, p \in H^{1}(\Omega) / \mathbb{R}$ such that

$$
\begin{gathered}
\mu(\mathbf{u}, \mathbf{v})_{\Omega_{P}}+\nu(\mathbf{c u r l} \mathbf{u}, \mathbf{c u r l} \mathbf{v})_{\Omega_{F}}+(\nabla p, \mathbf{v})=(\mathbf{f}, \mathbf{v}), \quad \forall \mathbf{v} \in X \\
-\mu(\mathbf{u}, \nabla q)_{\Omega_{F}}+(\nabla p, \nabla q)=(\mathbf{f}, \nabla q), \quad \forall q \in H^{1}(\Omega) / \mathbb{R} .
\end{gathered}
$$

\section{Finite element discretization}

In what follows and for simplicity, we make the further assumption that both $\Omega$ and $\Omega_{F}$ are polyhedra. We introduce a regular family of triangulation $\left(\tau_{h}\right)_{h}$ in the sense that:

- for each $h, \bar{\Omega}$ is the union of all elements of $\tau_{h}$;

- for each $h$, the intersection of two different elements of $\tau_{h}$, if not empty, is a corner, a whole edge or a whole face of both of them; 
- the ratio of the diameter $h_{\kappa}$ of an element $\kappa$ in $\tau_{h}$ to the diameter of its inscribed sphere is bounded by a constant independent of $\kappa$ and $h$;

As usual, $h$ denotes the maximum of the diameters of the elements of $\tau_{h}$. We denote by $\tau_{h}^{F}$ (resp. $\tau_{h}^{P}$ ) the set of elements $\kappa$ of $\tau_{h}$ which are contained in $\Omega_{F}$ (resp. $\Omega_{P}$ ).

Next, for each $\kappa$ in $\tau_{h}$, we introduce the spaces $\mathbb{P}_{0}(\kappa)$ of the restrictions to $\kappa$ of constant functions on $\mathbb{R}^{3}, \mathbb{P}_{1}(\kappa)$ of the restrictions to $\kappa$ of affine functions on $\mathbb{R}$ and the space $\mathbb{P}_{K}(\kappa)$ of the restrictions to $\kappa$ of polynomials $\mathbf{v}$ of the form:

$$
\mathbf{v}(\mathbf{x})=\mathbf{a}+\mathbf{b} \times \mathbf{x}, \quad \mathbf{a} \in \mathbb{R}^{3}, \mathbf{b} \in \mathbb{R}^{3} .
$$

The space $\mathbb{P}_{K}(\kappa)$ and the corresponding finite elements are studied in [17]. Their degrees of freedom are the average flux along the edges $\int_{l}(\mathbf{v} . \mathbf{t}) d l$, for the six edges $l$ of $\kappa, \mathbf{t}$ is the direction vector of $l$.

Hence, we associate the operator $r_{\kappa}$ where $r_{\kappa}(\mathbf{u})$ is the unique polynomial of $\mathbb{P}_{K}$ that has the same flux along the edges as $\mathbf{u}$. We define also the operator $I_{\kappa}$ where $I_{\kappa}(q)$ is the unique polynomial of $\mathbb{P}_{1}(\kappa)$ that has the same values on the vertex of $\kappa$ as $q$. We have for all $\kappa \in \tau_{h}$ :

Next, let us introduce the discrete spaces:

$$
r_{\kappa}(\nabla q)=\nabla I_{\kappa}(q) \quad \forall q \in W^{2, t}(\kappa) \quad \text { for some } t>2 .
$$

$$
\begin{aligned}
& X_{h}=\left\{\mathbf{u}_{h} \in X ; \mathbf{u}_{\left.h\right|_{\kappa}} \in \mathbb{P}_{K}(\kappa), \forall \kappa \in \tau_{h}\right\}, \\
& Q_{h}=\left\{q_{h} \in C^{0}(\bar{\Omega}) ; q_{\left.h\right|_{\kappa}} \in \mathbb{P}_{1}(\kappa), \forall \kappa \in \tau_{h}\right\},
\end{aligned}
$$

With these spaces, the finite dimensional analogues of $U$ is:

$$
U_{h}=\left\{\mathbf{v}_{h} \in X_{h} ;\left(\nabla q_{h}, \mathbf{v}_{h}\right)=0, \quad \forall q_{h} \in Q_{h}\right\},
$$

We define the interpolation operators $r_{h}$ from $H^{1}(\Omega)^{3}$ onto $X_{h}, I_{h}$ from $H^{2}(\Omega)$ onto $Q_{h}$ by

$$
\left.r_{h} u=r_{\kappa}(u) \text { on } \kappa, \forall \kappa \in \tau_{h} \quad \text { (similarly for } I_{h}\right) \text {. }
$$

We have the following result:

Theorem 3.1. Assume that $\tau_{h}$ is regular family of triangulations. We have:

$$
\left\|\mathbf{u}-r_{h} \mathbf{u}\right\|_{0, \Omega}+h\left\|\operatorname{curl}\left(\mathbf{u}-r_{h} \mathbf{u}\right)\right\|_{0, \Omega} \leq C h|\mathbf{u}|_{1, t, \Omega}, \forall \mathbf{u} \in W^{1, t}(\Omega)^{3}, \text { for some } t>2 \text {. }
$$

Moreover, when $\mathbf{u} \in H^{2}(\Omega)^{3}$, we have:

$$
\left\|\mathbf{u}-r_{h} \mathbf{u}\right\|_{0, \Omega} \leq C h^{2}|\mathbf{u}|_{2, \Omega}
$$

and

$$
\left\|\operatorname{curl}\left(\mathbf{u}-r_{h} \mathbf{u}\right)\right\|_{0, \Omega} \leq C h|\mathbf{u}|_{2, \Omega}
$$

Theorem 3.2. Let $\Omega$ be a polyhedron and $\Omega_{F}$ a convex polyhedron. Let $\tau_{h}$ be a uniformly regular family of triangulation of $\Omega$. We have:

$$
\left\|\mathbf{u}_{h}\right\|_{0, \Omega_{F}} \leq \alpha_{0}\left(\left\|\mathbf{u}_{h}\right\|_{0, \Omega_{P}}^{2}+\| \text { curl } \mathbf{u}_{h} \|_{0, \Omega_{F}}^{2}\right)^{1 / 2}, \quad \forall \mathbf{u}_{h} \in U_{h}
$$

Proof: The inequality (3.3) is valid for every $\mathbf{v} \in U$. Let $\Omega_{F}$ be convex, for every function $\mathbf{u}_{h}$ in $U_{h}$, we consider the Dirichlet problem:

$$
(\nabla z, \nabla \mu)_{\Omega_{F}}=\left(\mathbf{u}_{h}, \nabla \mu\right)_{\Omega_{F}} \quad \forall \mu \in H^{1}\left(\Omega_{F}\right) / \mathbb{R} .
$$

The difference $\mathbf{w}=\mathbf{u}_{h}-\nabla z$ belongs to the space

$$
U_{\Omega_{F}}=\left\{\mathbf{v} \in H\left(\operatorname{curl}, \Omega_{F}\right) ;(\mathbf{v}, \nabla q)_{\Omega_{F}}=0 \forall q \in H^{1}\left(\Omega_{F}\right) / \mathbb{R}\right\},
$$

and $\operatorname{curl} \mathbf{w}=$ curl $\mathbf{u}_{h}$. It follows from theorem 2.2 that

$$
\|\mathbf{w}\|_{1, \Omega_{F}} \leq C_{1} \| \text { curl w } \|_{0, \Omega_{F}} .
$$

Therefore, as curl $\mathbf{u}_{h} \in \mathrm{E}^{\infty}(\Omega)^{3}$, we have (see Nedelec [[17],[18]]) for $s>2$ :

$$
\|\mathbf{w}\|_{1, s, \Omega_{F}} \leq C_{2}\left\|\operatorname{curl} \mathbf{u}_{h}\right\|_{0, s, \Omega_{F}} .
$$


and

$$
\left\|r_{h} \mathbf{w}-\mathbf{w}\right\|_{0, \Omega_{F}} \leq C_{3}\left\|\mathbf{c u r l} \mathbf{u}_{h}\right\|_{0, \Omega_{F}} .
$$

Then, we can apply the interpolation operator $r_{h}$ to $\mathbf{w}$, and $\mathbf{u}_{h}$ splits into:

$$
\mathbf{u}_{h}=r_{h} \mathbf{w}+\nabla z_{h} \quad \text { with } z_{h} \in Q_{h} .
$$

Hence

Since on one hand

$$
\left\|\mathbf{u}_{h}\right\|_{0, \Omega_{F}} \leq\left\|r_{h} \mathbf{w}-\mathbf{w}\right\|_{0, \Omega_{F}}+\|\mathbf{w}\|_{0, \Omega_{F}}+\left\|\nabla z_{h}\right\|_{0, \Omega_{F}} .
$$

and on the other hand

$$
\left\|\mathbf{w}-r_{h} \mathbf{w}\right\|_{0, \Omega_{F}} \leq C_{3}\left\|\mathbf{c u r l} \mathbf{u}_{h}\right\|_{0, \Omega_{F}}
$$

$$
\|\mathbf{w}\|_{0, \Omega_{F}} \leq\|\mathbf{w}\|_{1, \Omega_{F}} \leq C_{1}\left\|\operatorname{curl} \mathbf{u}_{h}\right\|_{0, \Omega_{F}} .
$$

We see that it suffices to estimate $\left\|\nabla z_{h}\right\|_{0, \Omega_{F}}$. For all $\mu_{h} \in Q_{h}$ we have:

$$
\begin{aligned}
\left(\nabla z_{h}, \nabla \mu_{h}\right)_{\Omega_{F}} & =\left(\mathbf{u}_{h}-r_{h} \mathbf{w}, \nabla \mu_{h}\right)_{\Omega_{F}} \\
& =\left(\mathbf{u}_{h}, \nabla \mu_{h}\right)_{\Omega_{F}}+\left(\mathbf{w}-r_{h} \mathbf{w}, \nabla \mu_{h}\right)_{\Omega_{F}} \\
& =-\left(\mathbf{u}_{h}, \nabla \mu_{h}\right)_{\Omega_{P}}+\left(\mathbf{w}-r_{h} \mathbf{w}, \nabla \mu_{h}\right)_{\Omega_{F}} \\
& \leq\left\|\mathbf{u}_{h}\right\|_{0, \Omega_{P}}\left\|\nabla \mu_{h}\right\|_{0, \Omega_{P}}+C_{3}\left\|\mathbf{c u r l} \mathbf{u}_{h}\right\|_{0, \Omega_{F}}\left\|\nabla \mu_{h}\right\|_{0, \Omega_{F}}
\end{aligned}
$$

We choose $\mu_{h} \in Q_{h}$ such that $\left.\mu_{h}\right|_{\bar{\Omega}_{F}}=\left.z_{h}\right|_{\bar{\Omega}_{F}}$ and $\left.\mu_{h}\right|_{\partial \Omega}=\mathbf{0}$, and we obtain:

$$
\left\|\nabla \mu_{h}\right\|_{0, \Omega_{P}} \leq c_{1}\left\|z_{h}\right\|_{1 / 2, \Gamma} \leq c_{2}\left\|\nabla z_{h}\right\|_{0, \Omega_{F}}
$$

We deduce

$$
\left\|\nabla z_{h}\right\|_{0, \Omega_{F}} \leq C\left(\left\|\mathbf{u}_{h}\right\|_{0, \Omega_{P}}+\left\|\operatorname{curl} \mathbf{u}_{h}\right\|_{0, \Omega_{F}}\right)
$$

and finally the result.

We discretize $(V)$ by:

Find $\mathbf{u}_{h} \in U_{h}$ and $p_{h} \in Q_{h} / \mathbb{R}$ such that

$$
\mu\left(\mathbf{u}_{h}, \mathbf{v}_{h}\right)_{\Omega_{P}}+\nu\left(\operatorname{curl} \mathbf{u}_{h}, \operatorname{curl} \mathbf{v}_{h}\right)_{\Omega_{F}}+\left(\nabla p_{h}, \mathbf{v}_{h}\right)=\left(\mathbf{f}, \mathbf{v}_{h}\right) \quad \forall \mathbf{v}_{h} \in X_{h} .
$$

As in the continuous way, the last problem can be splited to

Find $\mathbf{u}_{h} \in U_{h}$ such that

$$
\mu\left(\mathbf{u}_{h}, \mathbf{v}_{h}\right)_{\Omega_{P}}+\nu\left(\operatorname{curl} \mathbf{u}_{h}, \operatorname{curl} \mathbf{v}_{h}\right)_{\Omega_{F}}=\left(\mathbf{f}, \mathbf{v}_{h}\right), \quad \forall \mathbf{v}_{h} \in U_{h},
$$

Find $p_{h} \in Q_{h} / \mathbb{R}$ such that

$$
\mu\left(\mathbf{u}_{h}, \nabla q_{h}\right)_{\Omega_{P}}+\left(\nabla p_{h}, \nabla q_{h}\right)=\left(\mathbf{f}, \nabla q_{h}\right), \quad \forall q_{h} \in Q_{h} / \mathbb{R} .
$$

Let $\Omega_{F}$ be convex, it is easy to show, using the theorem 3.2, that these two last discrete problems have a unique solution and we have:

$$
\left\|\mathbf{u}_{h}\right\|_{0, \Omega_{P}}+\left\|\mathbf{c u r l} \mathbf{u}_{h}\right\|_{0, \Omega_{F}} \leq C_{3}\|\mathbf{f}\|_{0, \Omega}
$$

and

$$
\left|p_{h}\right|_{1, \Omega} \leq C_{4}|| \mathbf{f} \|_{0, \Omega}
$$

It is obvious that the last problem is equivalent to:

Find $\mathbf{u}_{h} \in U_{h}$ and $p_{h} \in Q_{h} / \mathbb{R}$ such that

$$
\mu\left(\mathbf{u}_{h}, \mathbf{v}_{h}\right)_{\Omega_{P}}+\nu\left(\operatorname{curl} \mathbf{u}_{h}, \operatorname{curl} \mathbf{v}_{h}\right)_{\Omega_{F}}+\left(\nabla p_{h}, \mathbf{v}_{h}\right)=\left(\mathbf{f}, \mathbf{v}_{h}\right), \quad \forall \mathbf{v}_{h} \in X_{h},
$$

Find $p_{h} \in Q_{h} / \mathbb{R}$ such that

$$
-\mu\left(\mathbf{u}_{h}, \nabla q_{h}\right)_{\Omega_{F}}+\left(\nabla p_{h}, \nabla q_{h}\right)=\left(\mathbf{f}, \nabla q_{h}\right), \quad \forall q_{h} \in Q_{h} / \mathbb{R} .
$$




\section{A PRIORI ERROR ANALYSIS}

In this section, we will establish the error estimates for the pressure and the velocity. First of all, we consider the quantity $\mathbf{u}_{h}-r_{h} \mathbf{u}$ and we consider the finite dimensional problem:

Find $\lambda_{h} \in Q_{h} / \mathbb{R}$ such that

$$
\forall q_{h} \in Q_{h} / \mathbb{R}, \quad \int_{\Omega} \nabla \lambda_{h} \nabla q_{h}=\int_{\Omega}\left(\mathbf{u}_{h}-r_{h} \mathbf{u}\right) \nabla q_{h}
$$

which admits a unique solution $\lambda_{h}$ such that $\mathbf{w}_{h}=\left(\mathbf{u}_{h}-r_{h} \mathbf{u}\right)-\nabla \lambda_{h}$ is in the space $U_{h}$ with $\operatorname{curl}\left(\mathbf{u}_{h}-\right.$ $\left.r_{h} \mathbf{u}\right)=\operatorname{curl} \mathbf{w}_{h}$.

Furthermore we consider, for all $q_{h} \in Q_{h} / \mathbb{R}$, the relation

$$
\int_{\Omega} \nabla \lambda_{h} \nabla q_{h}=\int_{\Omega}\left(\mathbf{u}_{h}-r_{h} \mathbf{u}\right) \nabla q_{h}=-\int_{\Omega}\left(r_{h} \mathbf{u}-\mathbf{u}\right) \nabla q_{h}
$$

which gives by taking $q_{h}=\lambda_{h}$ and supposing that $\mathbf{u} \in H^{2}(\Omega)^{3}$ :

$$
\left|\lambda_{h}\right|_{1, \Omega} \leq C h^{2}\|\mathbf{u}\|_{2, \Omega}
$$

To obtain the a priori error estimate for the velocity, it suffices to show an error estimate of $\mathbf{w}_{h}$ and we conclude an error estimate of $\mathbf{u} \in H^{2}(\Omega)^{3}$ by using the theorem 3.2. Let $\Omega_{F}$ be convex. For all $\mathbf{u}_{h} \in U_{h}$, we have:

$$
\begin{aligned}
\left\|\mathbf{u}-\mathbf{u}_{h}\right\|_{X}^{2} & \leq\left\|\mathbf{u}-r_{h} \mathbf{u}\right\|_{0, \Omega}^{2}+\left\|r_{h} \mathbf{u}-\mathbf{u}_{h}\right\|_{0, \Omega}^{2}+\left\|\mathbf{c u r l}\left(\mathbf{u}-r_{h} \mathbf{u}\right)\right\|_{0, \Omega_{F}}^{2}+\left\|\mathbf{c u r l}\left(r_{h} \mathbf{u}-\mathbf{u}_{h}\right)\right\|_{0, \Omega_{F}}^{2} \\
& \leq C_{1}(u, \Omega)\left(h^{2}+\left\|r_{h} \mathbf{u}-\mathbf{u}_{h}\right\|_{0, \Omega}^{2}+\left\|\mathbf{c u r l}\left(r_{h} \mathbf{u}-\mathbf{u}_{h}\right)\right\|_{0, \Omega_{F}}^{2}\right) \\
& \leq C_{2}(u, \Omega)\left(h^{2}+\left\|\nabla \lambda_{h}\right\|_{0, \Omega}^{2}+\left\|\mathbf{w}_{h}\right\|_{0, \Omega}^{2}+\left\|\mathbf{c u r l} \mathbf{w}_{h}\right\|_{0, \Omega_{F}}^{2}\right) \\
& \leq C_{3}(u, \Omega)\left(h^{2}+\left\|\nabla \lambda_{h}\right\|_{0, \Omega}^{2}+\left\|\mathbf{w}_{h}\right\|_{0, \Omega_{P}}^{2}+\left\|\mathbf{c u r l} \mathbf{w}_{h}\right\|_{0, \Omega_{F}}^{2}\right)
\end{aligned}
$$

Next, to obtain the error estimate for $\mathbf{w}$, we consider the difference of the equation (2.2) with $\mathbf{v}=\mathbf{v}_{h} \in X_{h}$ and the equation (3.4):

$$
\mu \int_{\Omega_{P}}\left(\mathbf{u}-\mathbf{u}_{h}\right) \mathbf{v}_{h}+\nu \int_{\Omega_{F}} \operatorname{curl}\left(\mathbf{u}-\mathbf{u}_{h}\right) \operatorname{curl} \mathbf{v}_{h}+\int_{\Omega} \nabla\left(p-p_{h}\right) \mathbf{v}_{h}=0
$$

We insert $\pm r_{h} \mathbf{u}$ in the first and the second terms, $\pm I_{h} p$ in the third term and we obtain:

$$
\begin{aligned}
\mu \int_{\Omega_{P}}\left(r_{h} \mathbf{u}-\mathbf{u}_{h}, \mathbf{v}_{h}\right)+\nu \int_{\Omega_{F}} \operatorname{curl}\left(r_{h} \mathbf{u}-\mathbf{u}_{h}\right) \operatorname{curl} \mathbf{v}_{h}= \\
\quad \mu\left(r_{h} \mathbf{u}-\mathbf{u}, \mathbf{v}_{h}\right)_{\Omega_{P}}+\nu\left(\operatorname{curl}\left(r_{h} \mathbf{u}-\mathbf{u}_{h}\right), \operatorname{curl} \mathbf{v}_{h}\right)_{\Omega_{F}}-\left(\nabla\left(p-I_{h} p\right), \mathbf{v}_{h}\right)-\left(\nabla\left(I_{h} p-p_{h}\right), \mathbf{v}_{h}\right)
\end{aligned}
$$

We replace $\mathbf{u}_{h}-r_{h} \mathbf{u}=\nabla \lambda_{h}+\mathbf{w}_{h}$ and we choose $\mathbf{v}_{h}=\mathbf{w}_{h}$ to obtain:

$$
\begin{aligned}
\mu\left\|\mathbf{w}_{h}\right\|_{0, \Omega_{P}}^{2}+ & \nu\left\|\mathbf{c u r l} \mathbf{w}_{h}\right\|_{0, \Omega_{F}}^{2}= \\
& -\mu \int_{\Omega_{P}} \nabla \lambda_{h} \mathbf{w}_{h}+\mu\left(r_{h} \mathbf{u}-\mathbf{u}, \mathbf{w}_{h}\right)_{\Omega_{P}}+\nu\left(\operatorname{curl}\left(r_{h} \mathbf{u}-\mathbf{u}_{h}\right), \mathbf{c u r l} \mathbf{w}_{h}\right)_{\Omega_{F}}-\left(\nabla\left(p-I_{h} p\right), \mathbf{w}_{h}\right)
\end{aligned}
$$

By supposing that $p \in H^{2}(\Omega)$ and $\mathbf{u} \in H^{2}(\Omega)^{3}$, we deduce using the properties of $r_{h}$ and $I_{h}$, the formula $a . b \leq \frac{1}{2 \varepsilon} a^{2}+\frac{1}{2} \varepsilon b^{2}$ with a suitable choice of $\varepsilon$ and the previous upper bound of $\lambda$ :

$$
\mu\left\|\mathbf{w}_{h}\right\|_{0, \Omega_{P}}^{2}+\nu\left\|\mathbf{c u r l} \mathbf{w}_{h}\right\|_{0, \Omega_{F}}^{2} \leq C(\Omega) h^{2}\left(\|\mathbf{u}\|_{2, \Omega}^{2}+\|p\|_{2, \Omega}^{2}\right)
$$

Now, we will show an estimate for the pressure. We subtract the equation (2.5) with $q=q_{h} \in Q_{h} / \mathbb{R}$ from the equation (3.6) to get

$$
\mu \int_{\Omega_{P}}\left(\mathbf{u}-\mathbf{u}_{h}\right) \nabla q_{h}+\int_{\Omega} \nabla\left(p-p_{h}\right) \nabla q_{h}=0
$$

We insert $\pm I_{h} p$ in the second term and we choose $q_{h}=p_{h}-I_{h} p$ to obtain

$$
\left|p_{h}-I_{h} p\right|_{1, \Omega} \leq \mu \|\left.\left(\mathbf{u}-\mathbf{u}_{h}\right)\right|_{0, \Omega_{P}}+\left|p-I_{h} p\right|_{1, \Omega}
$$


We deduce the error estimate:

$$
\begin{aligned}
\left|p-p_{h}\right|_{1, \Omega} & \leq\left|p-I_{h} p\right|_{1, \Omega}+\left|I_{h} p-p_{h}\right|_{1, \Omega} \\
& \leq C(\Omega)\left(\left.|| p\right|_{2, \Omega}+\|\left.\mathbf{u}\right|_{2, \Omega}\right) h
\end{aligned}
$$

Theorem 4.1. If $\mathbf{u} \in H^{2}(\Omega)^{3}$ and $p \in H^{2}(\Omega)$, the theoretical solution ( $\left.\mathbf{u}, p\right)$ of the problem (2.4)-(2.5) and the numerical solution $\left(\mathbf{u}_{h}, p_{h}\right)$ of the problem (3.5)-(3.6) verify the error estimate:

$$
\| \mathbf{u}-\left.\mathbf{u}_{h}\right|_{X}+\left|p-p_{h}\right|_{1, \Omega} \leq C(\Omega, \mathbf{u}, p) h
$$

\section{A POSTERIORI ERROR ANALYSIS}

We now intend to prove a posteriori error estimates between the exact solution $(\mathbf{u}, p)$ of the problem (2.4)-(2.5) and the numerical solution $\left(\mathbf{u}_{h}, p_{h}\right)$ of the problem (3.7)-(3.8).

We first introduce the space

$$
Z_{h}=\left\{\mathbf{g}_{h} \in L^{2}(\Omega)^{3} ; \forall \kappa \in \tau_{h},\left.\mathbf{g}_{h}\right|_{\kappa} \in \mathbb{P}_{0}(\kappa)\right\}
$$

and we fix an approximation $\mathbf{f}_{h}$ of the data $\mathbf{f}$ in $Z_{h}$.

Next, we denote by $\varepsilon_{h}$ the set of all faces of the elements. For every element $\kappa$ in $\tau_{h}$, we denote by $\varepsilon_{\kappa}$ the set of faces of $\kappa$ that are not contained in $\Gamma, \Delta_{\kappa}$ (resp. $\Delta_{\kappa}^{F}$ or $\Delta_{\kappa}^{P}$ ) the set of union of elements of $\tau_{h}$ that intersect $\kappa$ (resp. contained in $\Omega_{F}$ or contained in $\Omega_{P}$ ), $\Delta_{e}$ (resp. $\Delta_{e}^{F}$ or $\Delta_{e}^{P}$ ) the union of elements of $\tau_{h}$ that intersect the face $e$ (resp. contained in $\Omega_{F}$ or contained in $\Omega_{P}$ ), $h_{\kappa}$ the diameter of $\kappa$ and $h_{e}$ the diameter of the face $e$. Also, $\mathbf{n}_{\kappa}$ stands for the unit outward normal vector to $\kappa$ on $\partial \kappa$ and $[\cdot]_{e}$ the jump through the face $e$ of $\kappa$. If the face $e$ is on $\Gamma,[\cdot]_{e}$ will be the trace on $e$ from the domain $\Omega_{F}$ or $\Omega_{P}$ containing $\kappa$, multiplied by 2 .

For the demonstration of the next theorems, we introduce for an element $\kappa$ of $\tau_{h}$, the bubble function $\psi_{\kappa}$ (resp. $\psi_{e}$ of the face $e$ ) which is equal to the product of the $d+1$ barycentric coordinates associated with the vertices of $\kappa$ (resp. of $e$ ) and $\mathcal{L}_{e}$ (resp. $\mathcal{L}_{e}^{F}$ or $\mathcal{L}_{e}^{P}$ ) the lifting operator from polynomials defined on $e$ into polynomials defined on the elements $\kappa$ and $\kappa^{\prime}$ containing $e$ (resp. elements contained in $\Omega_{F}$ or contained in $\Omega_{P}$ ), which is constructed by affine transformations from a fixed operator on the reference element.

Property 5.1. Denoting by $P_{r}(e)$ the polynomial of degrees $r$ on $e$, we have

$$
\forall v \text { of } P_{r}(e) \quad c\|v\|_{L^{2}(e)} \leq\left\|v \psi_{e}^{1 / 2}\right\|_{L^{2}(e)} \leq c^{\prime}\|v\|_{L^{2}(e)}
$$

and for any $v$ of $P_{r}(e)$ which vanishes on $\partial e$, we have

$$
\left\|\mathcal{L}_{e} v\right\|_{L^{2}(\kappa)}+h_{e}\left|\mathcal{L}_{e} v\right|_{H^{1}(\kappa)} \leq c h_{e}^{1 / 2}\|v\|_{L^{2}(e)} .
$$

We denote by $R_{h}$ the Clément operator [7]. For any function $q \in H_{0}^{1}(\Omega), R_{h} q \in Q_{h}$ verifies

$$
\begin{aligned}
& \left\|q-R_{h} q\right\|_{L^{2}(\kappa)} \leq c h_{\kappa}\|q\|_{H^{1}(\Delta \kappa)}, \\
& \left\|q-R_{h} q\right\|_{L^{2}(e)} \leq c h_{e}^{1 / 2}\|q\|_{H^{1}(\Delta e)} .
\end{aligned}
$$

Furthermore, we define $\rho_{h}$ as the $L^{2}$ projection of $z$ onto $Z_{0}$ such that, in each triangle $\kappa$ we have: for $z \in L^{2}(\Omega)$,

$$
\rho_{h}(z)=\frac{1}{|T|} \int_{\kappa} z(x) d x .
$$

We have the properties: $\forall \kappa \in \tau_{h}, \forall p \in H^{1}(\Omega)$,

$$
\begin{aligned}
& \| p-\left.\rho_{h} p\right|_{L^{2}(\kappa)} \leq c h_{\kappa}|p|_{1, \kappa} \\
& \| p-\left.\rho_{h} p\right|_{L^{2}(e)} \leq c h_{e}^{1 / 2}|p|_{1, \kappa}
\end{aligned}
$$


We also denote by $\mathcal{R}_{h}$ the Raviart-Thomas operator : for any smooth enough vectorial function $\mathbf{v}$ which is divergence-free on $\Omega, \mathcal{R}_{h} \mathbf{v}$ belongs to $X_{h}$ and satisfies

$$
\forall e \in \varepsilon_{h}, \quad \int_{e}\left(\mathbf{v}-\mathcal{R}_{\mathbf{h}} \mathbf{v}\right) \cdot \mathbf{n} d \tau=0 .
$$

Moreover, this operator satisfies, see [19]: $\forall \mathbf{v}$ in $H^{1}(\Omega)^{3}$ and $\forall \kappa$ in $\tau_{h}$,

$$
\begin{aligned}
& \left\|\mathbf{v}-\mathcal{R}_{h} \mathbf{v}\right\|_{L^{2}(\kappa)^{3}} \leq c h_{\kappa}\|\mathbf{v}\|_{H^{1}(\kappa)^{3}} \\
& \left\|\mathbf{v}-\mathcal{R}_{h} \mathbf{v}\right\|_{L^{2}(e)^{3}} \leq c h_{e}^{1 / 2}\|\mathbf{v}\|_{H^{1}(\Delta e)^{3}}
\end{aligned}
$$

To prove the a posteriori estimates, we begin by decomposing $\mathbf{u}-\mathbf{u}_{h}=\nabla \lambda+\mathbf{w}$ where $\lambda \in H^{1}(\Omega)$ and $\mathbf{w} \in U$. Then, we establish a posteriori estimate for $\lambda$ and $\mathbf{w}$ to deduce using the lemma (2.3)

$$
\begin{aligned}
\left\|\mathbf{u}-\mathbf{u}_{h}\right\|_{X}^{2} & =\|\nabla \lambda+\mathbf{w}\|_{0, \Omega}^{2}+\|\mathbf{c u r l} \mathbf{w}\|_{0, \Omega_{F}}^{2} \\
& \leq C\left(|\lambda|_{1, \Omega}^{2}+\|\mathbf{w}\|_{0, \Omega_{P}}^{2}+\|\mathbf{c u r l} \mathbf{w}\|_{0, \Omega_{F}}^{2}\right)
\end{aligned}
$$

and we finish with the a posteriori estimate for the pressure.

The error function $\mathbf{u}-\mathbf{u}_{h}$ belongs to $X$, there exists a unique solution $\lambda_{1} \in H^{1}\left(\Omega_{F}\right) / \mathbb{R}$ of the problem:

$$
\int_{\Omega_{F}} \nabla \lambda_{1} \nabla q=\int_{\Omega_{F}}\left(\mathbf{u}-\mathbf{u}_{h}\right) \nabla q \quad \forall q \in H^{1}\left(\Omega_{F}\right) / \mathbb{R}
$$

and the function $\mathbf{w}_{1}=\left(\mathbf{u}-\mathbf{u}_{h}\right)-\nabla \lambda_{1}$ belongs to $U_{F}=\left\{\mathbf{v} \in H\left(\mathbf{c u r l}, \Omega_{F}\right) ;(\mathbf{v}, \nabla q)_{\Omega_{F}}=0, \forall q \in H^{1}\left(\Omega_{F}\right)\right\}$. We define the function $\tilde{\mathbf{w}}$, equal to $\mathbf{w}_{1}$ in $\Omega_{F}$ and $\mathbf{0}$ in $\Omega_{P}$, which belongs to $U$ and verifies $\mathbf{c u r l} \tilde{\mathbf{w}}=\mathbf{c u r l w}_{1}$ in $\Omega_{F}$. Furthermore, there exists a unique solution $\lambda \in H^{1}(\Omega) / \mathbb{R}$ of the problem:

$$
\int_{\Omega} \nabla \lambda \nabla q=\int_{\Omega}\left(\mathbf{u}-\mathbf{u}_{h}\right) \nabla q \quad \forall q \in H^{1}(\Omega) / \mathbb{R},
$$

and the function $\mathbf{w}=\left(\mathbf{u}-\mathbf{u}_{h}\right)-\nabla \lambda$ belongs to $U$ and we have curlw $=$ curl $\mathbf{w}_{1}=\operatorname{curl}\left(\mathbf{u}-\mathbf{u}_{h}\right)$ in $\Omega_{F}$. We have, for all $q \in H^{1}(\Omega)$

$$
\begin{aligned}
\int_{\Omega} \nabla \lambda \nabla q & =\int_{\Omega}(\mathbf{w}+\nabla \lambda) \nabla q=\int_{\Omega}\left(\mathbf{u}-\mathbf{u}_{h}\right) \nabla q=-\int_{\Omega} \mathbf{u}_{h} \nabla q \\
& =-\int_{\Omega} \mathbf{u}_{h} \nabla\left(q-q_{h}\right)=-\frac{1}{2} \sum_{\kappa \in \tau_{h}}\left(\sum_{e \in \varepsilon_{\kappa}} \int_{e}\left[\mathbf{u}_{h} \cdot \mathbf{n}\right]\left(q-q_{h}\right)\right), \quad \forall q_{h} \in Q_{h} .
\end{aligned}
$$

We introduce the indicators

$$
\xi_{\kappa}=\sum_{e \in \varepsilon_{\kappa}} h_{e}^{1 / 2}\left\|\left[\mathbf{u}_{h} \cdot \mathbf{n}\right]\right\|_{0, e}
$$

Theorem 5.2. The following bounds hold

$$
\|\nabla \lambda\|_{0, \Omega} \leq C\left(\sum_{\kappa \in \tau_{h}} \xi_{\kappa}^{2}\right)^{1 / 2}
$$

and

$$
\xi_{\kappa} \leq c\|\nabla \lambda\|_{0, \Delta_{\kappa}}
$$

Proof: First we take, in the equation (5.3), $q=\lambda$ and $q_{h}=R_{h} q$, the image of $q$ by the Clément type regularization operator, and we obtain the upper bound. In order to find the lower bound, we take in the equation (5.3) $q_{h}=0$ and $q=\mathcal{L}_{e}\left(\left[\mathbf{u}_{h} \cdot \mathbf{n}\right] \psi_{e}\right)$, and we obtain

$$
\left\|\left[\mathbf{u}_{h} . \mathbf{n}\right]\right\|_{0, e} \leq C\left(h_{e}^{-1 / 2}|\lambda|_{1, \kappa \cup \kappa^{\prime}}\right)
$$

which leads to

$$
\xi_{\kappa} \leq c|\lambda|_{1, \Delta_{\kappa}} .
$$


To find a posteriori estimates for $\mathbf{w}$, we begin to establish upper and lower bounds for curl $\mathbf{w}$ in $\Omega_{F}$. We introduce the indicators

$$
\gamma_{\kappa, F}=h_{\kappa}\left\|\mathbf{f}_{h}-\nabla p_{h}\right\|_{0, \kappa}+\frac{\nu}{2} \sum_{e \in \varepsilon_{\kappa}} h_{\kappa}^{1 / 2}\left\|\left[\operatorname{curl} \mathbf{u}_{h} \times \mathbf{n}\right]\right\|_{0, e}, \text { if } \kappa \in \Omega_{F}
$$

Theorem 5.3. Let $\Omega_{F}$ be convex. The following bounds hold:

$$
\nu\|\mathbf{c u r l} \mathbf{w}\|_{0, \Omega_{F}} \leq C\left(\sum_{\kappa \in \tau_{h}^{F}} h_{\kappa}^{2}\left\|\mathbf{f}-\mathbf{f}_{h}\right\|_{0, \kappa}^{2}+\sum_{\kappa \in \tau_{h}^{F}} \gamma_{\kappa, F}^{2}\right)^{1 / 2}
$$

and

$$
\gamma_{\kappa, F} \leq c \sum_{e \in \varepsilon_{\kappa}}\left(\|\mathbf{c u r l} \mathbf{w}\|_{0, \Delta_{e}^{F}}^{2}+h_{e}^{2}\left|p-p_{h}\right|_{1, \Delta_{e}^{F}}^{2}+h_{e}^{2}\left\|\mathbf{f}-\mathbf{f}_{h}\right\|_{0, \Delta_{e}^{F}}^{2}\right)^{1 / 2}
$$

Proof : The error function $\mathbf{u}-\mathbf{u}_{h}$ verifies, by using the equations (2.2) and (3.4):

$\forall \mathbf{v} \in X, \forall \mathbf{v}_{h} \in X_{h}$

$$
\begin{aligned}
\mu \int_{\Omega_{P}}\left(\mathbf{u}-\mathbf{u}_{h}\right) \mathbf{v}+\nu \int_{\Omega_{F}} \operatorname{curl}\left(\mathbf{u}-\mathbf{u}_{h}\right) \operatorname{curl} \mathbf{v}+\int_{\Omega} \nabla\left(p-p_{h}\right) \mathbf{v} \\
=\mu \int_{\Omega_{P}}\left(\mathbf{u}-\mathbf{u}_{h}\right)\left(\mathbf{v}-\mathbf{v}_{h}\right)+\nu \int_{\Omega_{F}} \operatorname{curl}\left(\mathbf{u}-\mathbf{u}_{h}\right) \operatorname{curl}\left(\mathbf{v}-\mathbf{v}_{\mathbf{h}}\right)+\int_{\Omega} \nabla\left(p-p_{h}\right)\left(\mathbf{v}-\mathbf{v}_{h}\right) \\
=\left(\mathbf{f}, \mathbf{v}-\mathbf{v}_{h}\right)-\mu \int_{\Omega_{P}} \mathbf{u}_{h}\left(\mathbf{v}-\mathbf{v}_{h}\right)-\nu \int_{\Omega_{F}} \operatorname{curl} \mathbf{u}_{h} \operatorname{curl}\left(\mathbf{v}-\mathbf{v}_{\mathbf{h}}\right)-\int_{\Omega} \nabla p_{h}\left(\mathbf{v}-\mathbf{v}_{h}\right) \\
=\left(\left(\mathbf{f}-\mathbf{f}_{h}\right), \mathbf{v}-\mathbf{v}_{h}\right)+\int_{\Omega_{F}}\left(\mathbf{f}_{h}-\nabla p_{h}\right)\left(\mathbf{v}-\mathbf{v}_{h}\right)+\int_{\Omega_{P}}\left(\mathbf{f}_{h}-\nabla p_{h}-\mu \mathbf{u}_{h}\right)\left(\mathbf{v}-\mathbf{v}_{h}\right) \\
\quad-\nu \int_{\Omega_{F}} \operatorname{curl} \mathbf{u}_{h} \operatorname{curl}\left(\mathbf{v}-\mathbf{v}_{\mathbf{h}}\right)
\end{aligned}
$$

We replace $\mathbf{u}-\mathbf{u}_{h}$ by $\mathbf{w}+\nabla \lambda$, take $\mathbf{v}=\tilde{\mathbf{w}}$ and $\mathbf{v}_{h}=\mathcal{R}_{h} \mathbf{v}$, remark that $\operatorname{curl} \mathbf{w}=\mathbf{c u r l} \tilde{\mathbf{w}}$ in $\Omega_{F}$ and use the integration by part formula to obtain:

$$
\begin{aligned}
\nu\|\operatorname{curl} \mathbf{w}\|_{0, \Omega_{F}}^{2}= & \int_{\Omega_{F}}\left(\mathbf{f}-\mathbf{f}_{h}\right)\left(\tilde{\mathbf{w}}-\mathcal{R}_{h} \tilde{\mathbf{w}}\right)-\int_{\Omega_{F}}\left(\mathbf{f}_{h}-\nabla p_{h}\right)\left(\tilde{\mathbf{w}}-\mathcal{R}_{h} \tilde{\mathbf{w}}\right) \\
& -\frac{\nu}{2} \sum_{\kappa \in \tau_{h}^{F}} \sum_{e \in \varepsilon_{\kappa}} \int_{e}\left[\operatorname{curl} \mathbf{u}_{h} \times \mathbf{n}\right]\left(\tilde{\mathbf{w}}-\mathcal{R}_{h} \tilde{\mathbf{w}}\right)
\end{aligned}
$$

Since $\Omega_{F}$ is convex, the theorem 2.2 and the lemma 2.3 give:

$$
\begin{aligned}
\nu\|\mathbf{c u r l} \mathbf{w}\|_{0, \Omega_{F}} & \leq C\left(\sum_{\kappa \in \tau_{h}^{F}}\left(h_{\kappa}^{2}\left\|\mathbf{f}-\mathbf{f}_{h}\right\|_{0, \kappa}^{2}+h_{\kappa}^{2}\left\|\mathbf{f}_{h}-\nabla p_{h}\right\|_{0, \kappa}^{2}\right)^{1 / 2}+\frac{\nu}{2} \sum_{\kappa \in \tau_{h}^{F}} \sum_{e \in \varepsilon_{\kappa}} h_{\kappa}\left\|\left[\mathbf{c u r l} \mathbf{u}_{h} \times \mathbf{n}\right]\right\|_{0, e}^{2}\right)^{1 / 2} \\
& \leq C\left(\sum_{\kappa \in \tau_{h}^{F}} h_{\kappa}^{2}\left\|\mathbf{f}-\mathbf{f}_{h}\right\|_{0, \kappa}^{2}+\sum_{\kappa \in \tau_{h}^{F}} \gamma_{\kappa, F}^{2}\right)^{1 / 2}
\end{aligned}
$$

and we obtain the upper bound. For the lower bound, we choose in the equation (5.11), $\mathbf{v}_{h}=0$ and we take for an element $\kappa \in \Omega_{F}, \mathbf{v}=\left(\mathbf{f}_{h}-\nabla p_{h}\right) \psi_{\kappa}$ and remark that

$$
\int_{\kappa} \operatorname{curl} \mathbf{u}_{h} \operatorname{curl} \mathbf{v}=\int_{\partial \kappa}\left(\operatorname{curl} \mathbf{u}_{h} \times \mathbf{n}\right) \mathbf{v}=0
$$

to obtain using the inverse inequality $\|\mathbf{c u r l} \mathbf{v}\|_{0, \kappa} \leq h_{\kappa}^{-1}\|\mathbf{v}\|_{0, \kappa}$ :

$$
\left\|\mathbf{f}_{h}-\nabla p_{h}\right\|_{0, \kappa}^{2} \leq\left\|\mathbf{f}-\mathbf{f}_{h}\right\|_{0, \kappa}^{2}+\left|p-p_{h}\right|_{1, \kappa}^{2}+h_{\kappa}^{-2}\|\mathbf{c u r l} \mathbf{w}\|_{0, \kappa}^{2}
$$

Next, we take $\mathbf{v}=\left.\mathcal{L}_{e}\left(\left[\operatorname{curl} \mathbf{u}_{h} \times \mathbf{n}\right] \psi_{e}\right)\right|_{\Omega_{F}}$ and integrate by part the last term of the equation (5.11) to obtain

$\nu\left\|\left[\mathbf{c u r l} \mathbf{u}_{h} \times \mathbf{n}\right] \psi_{e}^{1 / 2}\right\|_{0, e} \leq c\left(h_{e}^{-1 / 2}\|\mathbf{c u r l} \mathbf{w}\|_{0, \Delta_{e}^{F}}+h_{e}^{1 / 2}\left|p-p_{h}\right|_{1, \Delta_{e}^{F}}+h_{e}^{1 / 2}\left\|\mathbf{f}_{h}-\nabla p_{h}\right\|_{0, \Delta_{e}^{F}}+h_{e}^{1 / 2}\left\|\mathbf{f}-\mathbf{f}_{h}\right\|_{0, \Delta_{e}^{F}}\right)$ 
and we deduce the lower bound.

Before showing bounds on $\mathbf{w}$, we need to show bounds on $p-p_{h}$ in $\Omega_{F}$. For $\kappa \in \Omega_{F}$, we introduce the indicator:

$$
\eta_{\kappa, F}=\sum_{e \in \varepsilon_{\kappa}} h_{e}^{1 / 2}\left\|\left(\mathbf{f}_{h}-\nabla p_{h}\right) \cdot \mathbf{n}\right\|_{L^{2}(e)}
$$

Theorem 5.4. The pressure error verifies the bounds:

$$
\left|p-p_{h}\right|_{1, \Omega_{F}} \leq C\left(\sum_{\kappa \in \tau_{h}} \eta_{\kappa, F}^{2}+\sum_{\kappa \in \tau_{h}}\left\|\mathbf{f}-\mathbf{f}_{h}\right\|_{L^{2}(\kappa)}^{2}\right)^{1 / 2}
$$

and

$$
\eta_{\kappa, F} \leq c \sum_{e \in \varepsilon_{\kappa}}\left(\left\|\mathbf{f}-\mathbf{f}_{h}\right\|_{L^{2}\left(\Delta_{e}^{F}\right)}+\left\|\nabla\left(p-p_{h}\right)\right\|_{L^{2}\left(\Delta_{e}^{F}\right)}\right)
$$

Proof: For all $q \in H^{1}\left(\Omega_{F}\right)$, we take the second equation of the system $(P)$, multiply by $\nabla q$, integrate in $\Omega_{F}$ and obtain:

$$
\int_{\Omega_{F}} \nabla p \nabla q=\int_{\Omega_{F}} \mathbf{f} \nabla q
$$

Then we have by considering the definition of $\rho_{h}$ and by integrating by parts:

$$
\begin{array}{rlrl}
\int_{\Omega_{F}} \nabla\left(p-p_{h}\right) \nabla q & =\int_{\Omega_{F}}\left(\mathbf{f}-\mathbf{f}_{h}\right) \nabla q+\int_{\Omega_{F}}\left(\mathbf{f}_{h}-\nabla p_{h}\right) \nabla q & \\
& =\sum_{\kappa \in \tau_{h}^{F}}\left\{\int_{\kappa}\left(\mathbf{f}-\mathbf{f}_{h}\right) \nabla q+\int_{\kappa}\left(\mathbf{f}_{h}-\nabla p_{h}\right) \nabla\left(q-q_{h}\right)\right\}, \quad \forall q_{h} \in Z_{h} \\
& =\sum_{\kappa \in \tau_{h}^{F}}\left\{\int_{\kappa}\left(\mathbf{f}-\mathbf{f}_{h}\right) \nabla q+\sum_{e \in \varepsilon_{\kappa}} \int_{e}\left(\left(\mathbf{f}_{h}-\nabla p_{h}\right) \cdot \mathbf{n}\right)\left(q-q_{h}\right)\right\} &
\end{array}
$$

which leads, by taking $q=p-p_{h}, q_{h}=\rho_{h}(q)$ and using the properties of $\rho_{h}$, to (5.15). In the last equation and for every element $\kappa \in \Omega_{F}$, we take $q_{h}=0$ and $q=\mathcal{L}_{e}\left(\left[\left(\mathbf{f}_{h}-\nabla p_{h}\right) \cdot \mathbf{n}\right] \psi_{e}\right)$ to obtain (5.16).

To complete the upper and lower bounds of the velocity error, we show bound on $\mathbf{w}$ in $\Omega_{P}$. We introduce the indicators

$$
\gamma_{\kappa, P}=\left\|\mathbf{f}_{h}-\nabla p_{h}-\mu \mathbf{u}_{h}\right\|_{0, \kappa} \text { if } \kappa \in \Omega_{P}
$$

Theorem 5.5. The following bounds hold:

$$
\mu\|\mathbf{w}\|_{0, \Omega_{P}} \leq C\left(\left(\sum_{\kappa \in \tau_{h}^{P}}\left\|\mathbf{f}-\mathbf{f}_{h}\right\|_{0, \kappa}^{2}\right)^{1 / 2}+\left(\sum_{\kappa \in \tau_{h}^{F}} \gamma_{\kappa, P}^{2}\right)^{1 / 2}+\mu\|\nabla \lambda\|_{L^{2}\left(\Omega_{P}\right)}+\left\|\nabla\left(p-p_{h}\right)\right\|_{L^{2}\left(\Omega_{F}\right)}\right)
$$

and

$$
\gamma_{\kappa, P} \leq C\left(\left\|\mathbf{f}-\mathbf{f}_{h}\right\|_{0, \kappa}^{2}+\|\mathbf{w}\|_{0, \kappa}^{2}+\left|p-p_{h}\right|_{1, \kappa}^{2}+|\lambda|_{1, k}^{2}\right)^{1 / 2}
$$

Proof : In the equation (5.11), we replace $\mathbf{u}-\mathbf{u}_{h}=\mathbf{w}+\nabla \lambda$ and we take $\mathbf{v}=\mathbf{w}$ in $\Omega_{P}$ and $\mathbf{0}$ in $\Omega_{F}$. We choose $\mathbf{v}_{h}=0$ and we obtain:

$$
\mu\|\mathbf{w}\|_{0, \Omega_{P}}^{2}=\int_{\Omega_{P}}\left(\mathbf{f}-\mathbf{f}_{h}\right) \mathbf{w}+\int_{\Omega_{P}}\left(\mathbf{f}_{h}-\nabla p_{h}-\mu \mathbf{u}_{h}\right) \mathbf{w}-\mu \int_{\Omega_{P}} \mathbf{w} \nabla \lambda-\int_{\Omega_{P}} \nabla\left(p-p_{h}\right) \mathbf{w}
$$

Furthermore, we have:

$$
\begin{aligned}
\left|\int_{\Omega_{P}} \nabla\left(p-p_{h}\right) \mathbf{w}\right| & =\left|\int_{\Gamma} \mathbf{w} \cdot \mathbf{n}\left(p-p_{h}\right)\right| \leq\|\mathbf{w} \cdot \mathbf{n}\|_{-1 / 2, \Gamma}\left\|p-p_{h}\right\|_{1 / 2, \Gamma} \\
& \leq\|\mathbf{w}\|_{H\left(\operatorname{div}, \Omega_{p}\right)}\left\|\nabla\left(p-p_{h}\right)\right\|_{0, \Omega_{F}}=\|\mathbf{w}\|_{0, \Omega_{p}}\left\|\nabla\left(p-p_{h}\right)\right\|_{0, \Omega_{F}}
\end{aligned}
$$

Then we get the upper bound (5.18). For the lower bound, we choose $\mathbf{v}_{h}=0$ in the equation (5.11) and we take for an element $\kappa \in \Omega_{P}, \mathbf{v}=\left(\mathbf{f}_{h}-\nabla p_{h}-\mu \mathbf{u}_{h}\right) \psi_{\kappa}$ to obtain the lower bound. 
To show an upper and a lower bound of the pressure, we define the indicators:

$$
\eta_{\kappa}= \begin{cases}\eta_{\kappa, F} & \text { if } \kappa \in \Omega_{F} \\ \eta_{\kappa, P}=\sum_{e \in \varepsilon_{\kappa}} h_{e}^{1 / 2}\left\|\left(\mathbf{f}_{h}-\nabla p_{h}-\mu \mathbf{u}_{h}\right) \cdot \mathbf{n}\right\|_{L^{2}(e)} & \text { if } \kappa \in \Omega_{P}\end{cases}
$$

Theorem 5.6. The following bounds hold

$$
\left|p-p_{h}\right|_{1, \Omega_{P}} \leq C\left\{\mu|| \mathbf{w}\left\|_{0, \Omega_{P}}^{2}+\mu \mid\right\| \nabla \lambda \|_{0, \Omega_{P}}^{2}+\sum_{\kappa \in \tau_{h}^{F}}\left(\eta_{\kappa}^{2}+\left\|\mathbf{f}-\mathbf{f}_{h}\right\|_{L^{2}(\kappa)}^{2}\right)\right\}^{1 / 2}
$$

and

$$
\eta_{\kappa, P} \leq C\left(\|\nabla \lambda\|_{0, \Delta_{\kappa}^{P}}+\|\mathbf{w}\|_{0, \Delta_{\kappa}^{P}}+\left|p-p_{h}\right|_{H^{1}\left(\Delta_{\kappa}^{P}\right)}+\left\|\mathbf{f}-\mathbf{f}_{h}\right\|_{L^{2}\left(\Delta_{\kappa}^{P}\right)}\right)
$$

Proof : The error function $p-p_{h}$ belongs to $H^{1}(\Omega)$ and satisfies, using the first equation of the system $(\mathrm{P})$, for all $q \in H^{1}(\Omega)$ :

$$
\mu\left(\mathbf{u}-\mathbf{u}_{h}, \nabla q\right)_{\Omega_{P}}+\left(\nabla\left(p-p_{h}\right), \nabla q\right)_{\Omega_{P}}=\mu\left(\mathbf{f}-\mathbf{f}_{h}, \nabla q\right)_{\Omega_{P}}+\left(\mathbf{f}_{h}-\nabla p_{h}-\mu \mathbf{u}_{h}, \nabla q\right)_{\Omega_{P}}
$$

We replace $\mathbf{u}-\mathbf{u}_{h}$ by $\nabla \lambda+\mathbf{w}$ and we obtain for all $q \in H^{1}(\Omega)$ and $q_{h} \in Z_{h}$ :

$$
\begin{aligned}
\left(\nabla\left(p-p_{h}\right), \nabla q\right)_{\Omega_{P}}= & \int_{\Omega_{P}}\left(\mathbf{f}-\mathbf{f}_{h}\right) \nabla q-\mu \int_{\Omega_{P}} \nabla \lambda \nabla q-\mu \int_{\Omega_{P}} \mathbf{w} \nabla q \\
& +\int_{\Omega_{P}}\left(\mathbf{f}_{h}-\nabla p_{h}-\mu \mathbf{u}_{h}\right) \nabla\left(q-q_{h}\right)
\end{aligned}
$$

Integrating by part, taking $q=p-p_{h}$ and $q_{h}=\rho_{h}(q)$, remarking that $\operatorname{div} \mathbf{f}_{h}=\operatorname{div} \mathbf{u}_{h}=\operatorname{div} \nabla p_{h}=0$ in every element $\kappa \in \tau_{h}$ and using the properties of $\rho_{h}$, we obtain the upper bound (5.22).

To prove the inequality (5.23), we take $q_{h}=0$, integrate by part the last term and take $q=\mathcal{L}_{e}^{P}\left(\left(\mathbf{f}_{h}-\right.\right.$ $\left.\left.\left.\nabla p_{h}-\nu \mathbf{u}_{h}\right) . \mathbf{n} \psi_{e}\right)\right)$ in the equation (5.25) to obtain the inequality (5.23).

To simplify the notations, we define the indicators:

$$
\gamma_{\kappa}= \begin{cases}\gamma_{\kappa, F} & \text { if } \kappa \in \Omega_{F} \\ \gamma_{\kappa, P} & \text { if } \kappa \in \Omega_{P}\end{cases}
$$

Corollary 5.7. Let $\Omega_{F}$ be convex. The optimal a posteriori estimate holds

$$
\left\|\mathbf{u}-\mathbf{u}_{h}\right\|_{X}+\left|p-p_{h}\right|_{1, \Omega} \leq\left\{\sum_{\kappa \in \tau_{h}}\left(\gamma_{\kappa}^{2}+\xi_{\kappa}^{2}+\eta_{\kappa}^{2}+\left\|\mathbf{f}-\mathbf{f}_{h}\right\|_{0, \kappa}^{2}\right)\right\}^{1 / 2}
$$

where $\xi_{\kappa}, \gamma_{\kappa}$ and $\eta_{\kappa}$ are given by the formulas (5.6), (5.10), (5.19), (5.16) and (5.23).

Conclusion: We observe that estimate (5.26) is optimal: up to the terms involving the data, the full error is bounded by a constant times the sum of all indicators. Estimates (5.6), (5.10), (5.19), (5.16) and (5.23) are local, i.e., only involve the error in a neighborhood of K or e. The indicators $\eta_{\kappa}, \xi_{\kappa}$ and $\gamma_{\kappa}$ can be viewed as a measure for the error of the space discretization and can be used to adapt the mesh-size in space.

\section{Numerical Results}

To validate the theoretical results, we performed several numerical simulations using the FreeFem ++ software (see [15]). The geometry considered is a sphere centered at the origin of radius 0.5 , contained in the cubic domain $]-1,1[\times]-1,1[\times]-1,1[$. The numerical velocity and the pressure are taken as $(\mathbf{u}, p)=(\operatorname{curl} \psi, p)$, where:

$$
\begin{gathered}
\psi=(\varphi, \varphi, \varphi) \text { with } \varphi(x, y, z)=(x-1)^{2}(x+1)^{2}(y-1)^{2}(y+1)^{2}(z-1)^{2}(z+1)^{2}\left(x^{2}+y^{2}+z^{2}-0.25\right)^{3}, \\
\text { and } p(x, y, z)=-x-y-z .
\end{gathered}
$$


We take $\mu$ and $\nu$ equal to 1 . To get the numerical solution of the problem (3.4), we use, for some small parameter $\varepsilon$, the modified problem

Find $\mathbf{u}_{h} \in X_{h}$ and $p_{h} \in Q_{h} / \mathbb{R}$ such that

$$
\begin{array}{ll} 
& \left(\mathbf{u}_{h}, \mathbf{v}_{h}\right)_{\Omega_{P}}+\left(\operatorname{curl} \mathbf{u}_{h}, \operatorname{curl} \mathbf{v}_{h}\right)_{\Omega_{F}}+\left(\nabla p_{h}, v_{h}\right)=\left(\mathbf{f}, \mathbf{v}_{h}\right), \quad \forall \mathbf{v}_{h} \in X_{h}, \\
\text { and } \quad\left(\mathbf{u}_{h}, \nabla q_{h}\right)+\varepsilon\left(p_{h}, q_{h}\right)=0, \quad \forall q_{h} \in Q_{h} / \mathbb{R} .
\end{array}
$$

Indeed, in comparison with problem (3.4), we have added the term $\left(p_{h}, q_{h}\right)$ multiplied by $\varepsilon$ to obtain an invertible matrix. We have checked that there is no dependency of the solution upon $\varepsilon$, wich is fixed here equal to $10^{-10}$.

In what follows, we present the results obtained for the "a priori" part. The geometry mesh is given by figure 2 .

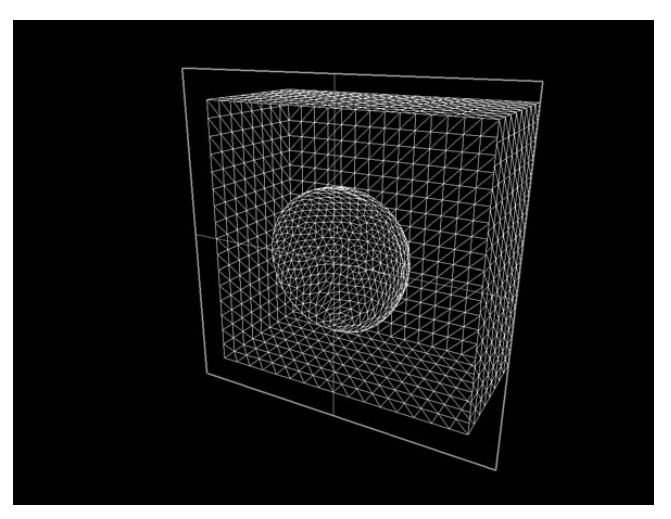

FiguRE 2. The a priori geometry mesh

In figure 3 , we can see a comparison between the theoretical velocity $\mathbf{u}$ and the numerical one $\mathbf{u}_{h}$, for some random degrees of freedom (left figure) as well as a more precise vue of the behavior of $u$ and $u_{h}$.
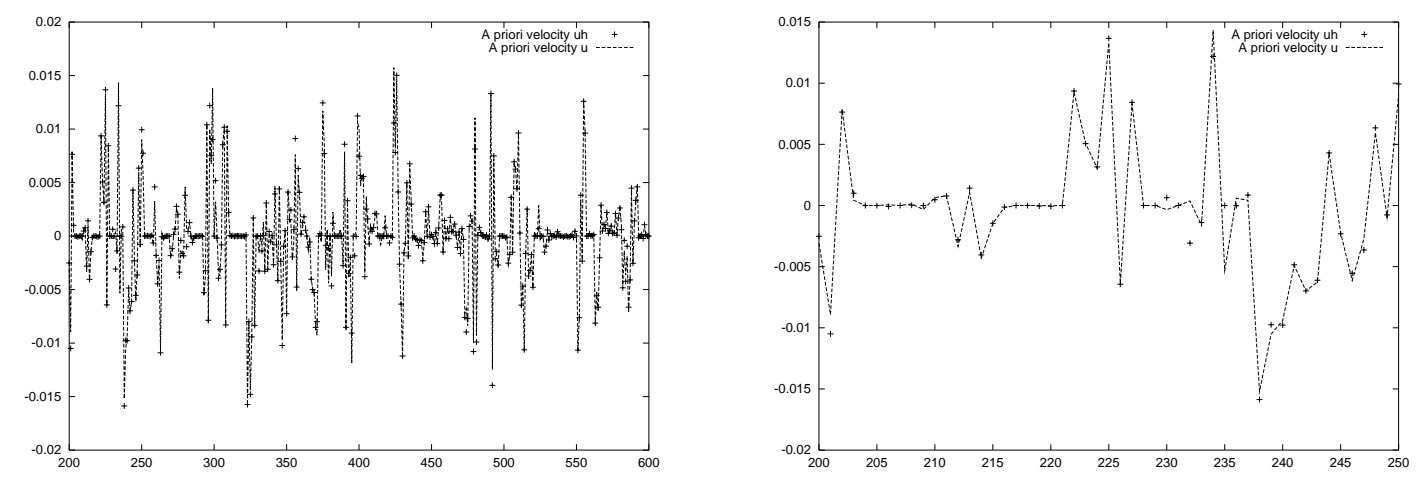

Figure 3 . The left figure represent a comparison between the exact and the numerical velocity for the "a priori" part. The right figure represent a precise vue of $u$ and $u_{h}$ 
Finally, curves displayed in figure 4 describe the errors $\left\|\mathbf{u}-\mathbf{u}_{h}\right\|_{X}$ and $\left|p-p_{h}\right|_{1, \Omega}$ in logarithmic scale.
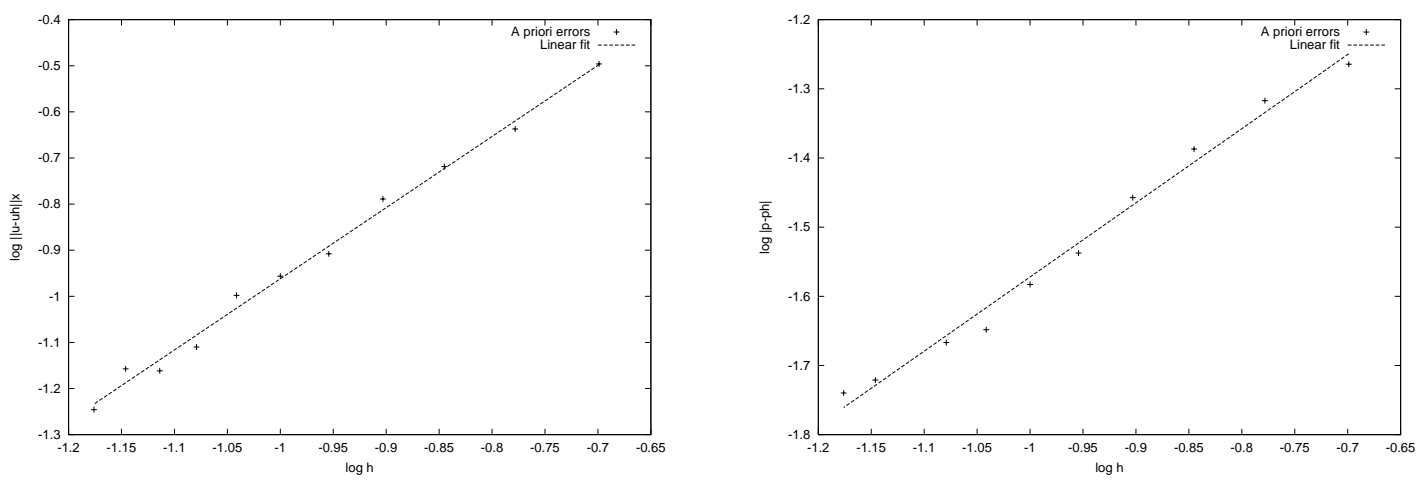

FIGURE 4. The left and right graphs represent respectively the a priori error of the velocity and the pressure

We can see that the pressure slope is 1.072 and the velocity slope is 1.543 . These results confirm the theoretical ones.

Concerning the a posteriori results, we generate new adapted meshes (see [13] and [14], for instance) and we consider a different velocity on $\Omega_{F}$ and $\Omega_{P}$, the pressure remains the same. Indeed, we take

$$
\varphi(x, y, z)=x^{2} y^{2} z^{2} \text { in } \Omega_{F},
$$$$
\text { and } \varphi(x, y, z)=(x-1)^{2}(x+1)^{2}(y-1)^{2}(y+1)^{2}(z-1)^{2}(z+1)^{2}\left(x^{2}+y^{2}+z^{2}\right) \text { in } \Omega_{P} .
$$

Figure 5 shows the considered adapted mesh. We can note that the geometry is more refined outside the sphere rather than inside it, where the solution is more smooth.

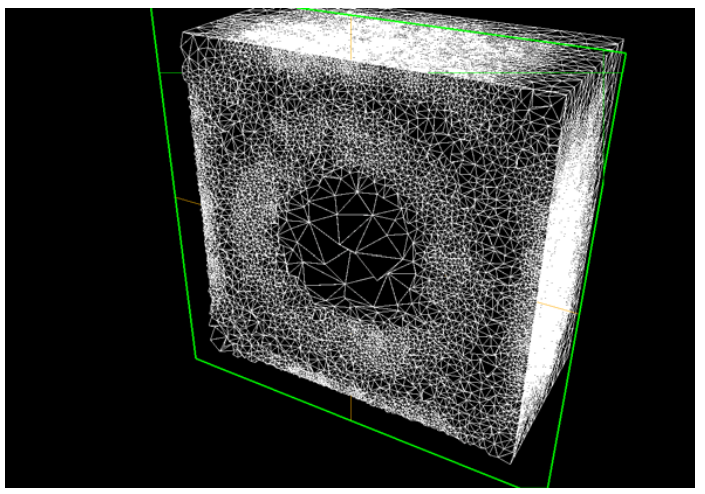

FIGURE 5. The a posteriori adapted mesh 
In figures 6 and 7, we present a comparison between the initial mesh and the adapted one for the velocity and the pressure respectively.
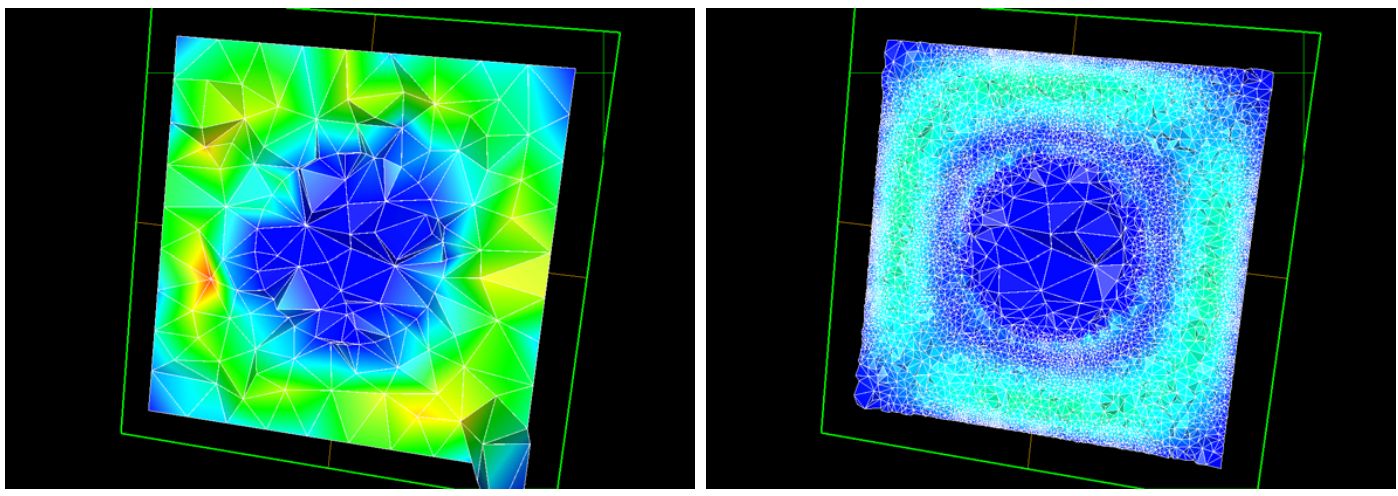

FIGURE 6 . The left and right figures represent respectively the velocity before and after the adapted mesh
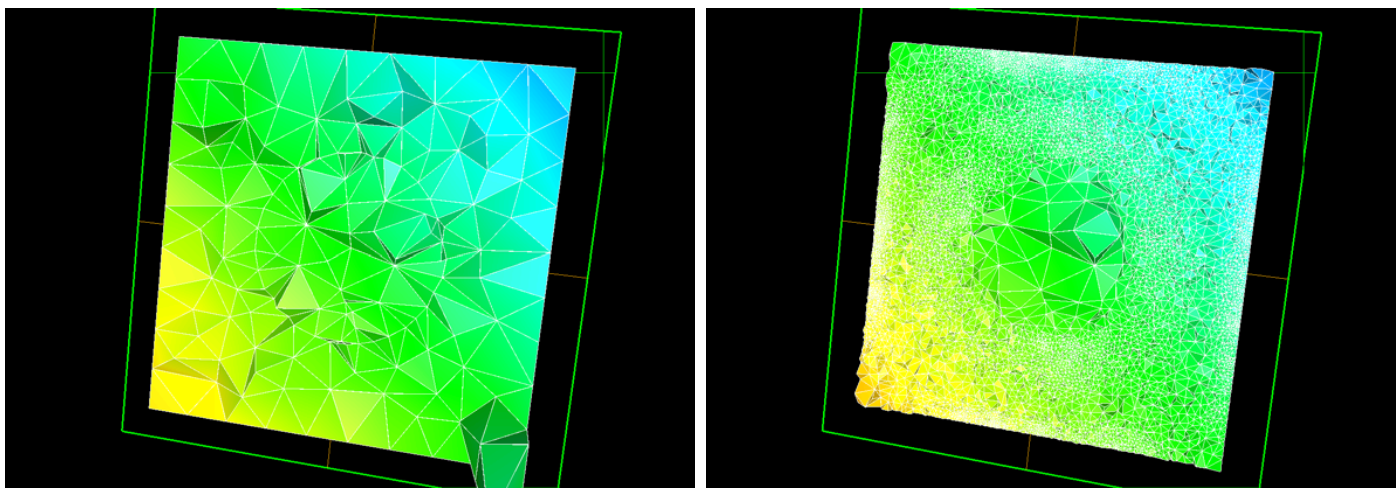

FiguRE 7. The left and right figures represent respectively the pressure before and after the adapted mesh

\section{REFERENCES}

[1] H. Abboud, F. El Chami \& T. Sayah, A priori and a posteriori estimates for three dimentional Stokes equations with non standard boundary conditions, submited.

[2] T. Arbogast \& D.S. Brunson A computational method for approximating a Darcy-Stokes system governing a vuggy porous medium, Comput. Geosci., Vol. 11, pp. 207-218 (207).

[3] M. L. Badea, M. Discacciati \& A. Quarteroni Mathematical analysis of the Navier-Stokes/Darcy coupling, Numer. Math. 115(2), 195-227 (2010).

[4] C. Bernardi, F. Hecht, O. Pironneau Coupling Darcy and Stokes Equations for the porous media with craks, ESAIM: M2AN, Vol. 39, No 1, 2005, pp. 7-35.

[5] C. Bernardi, F. Hecht \& Z. Mghazli Mortar finite element discretization for the flow in a non homogeneous porous medium, Comput. Methods Appl. Mech. Engrg., Vol. 196, 2007, pp. 1554-1573.

[6] C. Bernardi, F. Hecht \& Fatma Zohra Nouri A new finite element discretization of the Stokes problem coupled with Darcy equations, IMA Journal of Numerical Analysis, Vol. 30, 2010, pp. 61-93.

[7] P. Clément Approximation by finite element functions using local regularisation.,R.A.I.R.O. Anal. Numer. 9,pp, 77$84(1975)$

[8] M. Discacciati \& A. Quarteroni Analysis of a domain decomposition method for the coupling of Stokes and Darcy equations, In Numerical Mathematics and Advanced Applications - ENUMATH 2001, F. Brezzi et al, eds, p. 3-20, Springer, Milan (2003).

[9] M. Discacciati \& A. Quarteroni Convergence analysis of a subdomain iterative method for the finite element approximation of the coupling of Stokes and Darcy equations, Comput. Vis. Sci. 6(2-3), 93-103 (2004).

[10] F. Dubois. Vorticity-velocity-pressure formulation for the Stokes problem, Math. Meth. in the Applied Sciences, 25 (2002), 1091-1119. 
[11] F. Dubois, M. Salaün \& S. Salmon. Vorticity-velocity-pressure and stream function-vorticity formulations for the Stokes problem, J. Math. Pures Appl., 82 (2003), 1395-1451.

[12] F. El Chami \& T. SAYAh, A posteriori error estimators for the fully discrete time dependent Stokes problem with some different boundary conditions, CALCOLO, 47 (3) 2010, 169-192.

[13] P.J. Frey \& P.-L. George, Maillages, applications aux Â'elÂ'ements finis, Herm'es, Paris, (1999).

[14] P.-L. George \& F. Несht, Non-isotropic grids. Handbook of Grid Generation, J.F. Thompson, B.K. Soni and N.P. Weatherhill Eds., CRC Press, (1998).

[15] F. Hecht \& O. Pironneau, FreeFem++, see: http://www.freefem.org.

[16] M. MU \& J. XU A two-grid method of a mixed Stokes-Darcy model for coupling fluid flow with porous media flow, SIAM J. Numer. Anal. (2007), Vol. 45, No. 5, pp. 1801-1813 (2007).

[17] J. C. NedeleC Mixed finite element in $\mathbb{R}^{3}$. Numer. Math. 35, pp. 315-341 (1980).

[18] J. C. Nedelec Eléments finis mixtes incompressibles pour l'équation de Stokes dans $\mathbb{R}^{3}$. Numer. Math. 39, pp. 97-112 (1982).

[19] P.-A. Raviart \& J.-M. Thomas A mixed finite element method for second order elliptic problems, Mathematical Aspects of Finite Element Methods. Springer, Berlin. Lect. Notes Math., 606 pp 292-315 (1977).

[20] S. SALmon Développement numérique de la formulation tourbillon-vitesse-pression pour le problème de Stokes, Thesis, Université Pierre et Marie Curie, Paris (1999).

[21] B. Rivière \& I. Yotov Locally conservative coupling of Stokes and Darcy flows, SIAM J. Numer. Anal., Vol. 40, No. 5, pp. 1959-1977 (2005). 